\title{
LO SCRIPTORIUM DI SAN NICOLA DI CASOLE (OTRANTO, LECCE) E IL SUO TYPIKON (CODEX TAURINENSIS GRAECUS 216): UN'ANALISI STORICO-LETTERARIA
}

\section{MATTIA COSIMO CHIRIATTI}

\author{
UDC: $27-788(450.758) " 11 "$ \\ 7.071.3 \\ Original scientific paper
}

Manuscript received: 01. 11. 2016.

Revised manuscript accepted: 18. 02. 2017.

DOI: 10.1484/J.HAM.5.113734

\author{
M. C. Chiriatti \\ Grup de Recerques en Antiguitat Tardana \\ Departament d'Història i Arqueologia \\ Universidad de Barcelona \\ Montalegre, 108001 Barcelona \\ España
}

A typikon, stored in the library of the University of Turin under the signature Graecus 216 (ex-Royal Library Codex C III 17), portrays the industrious cultural activity of the monastery of St. Nicholas of Kasoulon, founded in 1098/99 by the monk Joseph, thanks to the patronage of Bohemund, prince of Taranto and Antioch, about a few miles south of Otranto (Lecce), in the south-eastern tip of Italy. According to the testimony of the manuscript, it can be given a broad outline of the scriptorium's daily activity as a crucial centre of culture and knowledge's transmission through the manuscripts' copying. This factor allowed a considerable diffusion of texts both in the religious either in the secular milieu, by making the Hydruntine coenobium to become one of the Byzantine culture's most outstanding centres in Southern Italy.

Keywords: Typikon, St. Nicholas of Kasoulon, Scriptorium, Manuscripts, Byzantine.

\section{INTRODUZIONE}

"Dopo di esso ecco il cenobio dedicato a San Nicola, un miglio e mezzo lontano da Otranto. Qui viveva una numerosa comunità di monaci basiliani, assolutamente meritevoli di venerazione, istruiti tutti nella conoscenza delle lettere greche e moltissimi anche in quella delle lettere latine, che offriva all'esterno un'eccellente immagine di sé. A quanti volessero apprendere le lettere greche, essi assicuravano la maggior parte del vitto, un insegnante e ospitalità senza richiedere alcun compenso. In tal modo si sosteneva lo studio del greco e si alimentava la comprensione della cultura greca che oggi, invece, fanno registrare un arretramento. Al tempo dei miei antenati, quando ancora esisteva la reggia di Costantinopoli, vi fu un filosofo, Nicola d'Otranto, di cui, prima della venuta dei turchi, si conservavano in questo monastero molte opere di logica e di filosofia. Costui, creato abbate di questa comunità di religiosi e assunto il nome di Niceta, spesso si recava dal sommo pontefice all'imperatore e da quello al sommo pontefice per mediare rispettive posizioni quando tra il pontefice el'imperatore insorgeva qualche motivo di dissenso riguardante o l'ortodossia della fede o altro argomento. Era infatti figura di grandissima autorevolezza e persona dai costumi assolutamente irreprensibili, come è proprio di chi dallo studio e dalla pratica filosofica era passato alla vita religiosa. Egli, senza badare a spese, costituì in questo cenobio una biblioteca che raccoglieva ogni genere di libri, quanti ne poté rintracciare per tutta la Grecia. Di questi una gran parte andò dispersa per la colpevole negligenza dei Latini, che disprezzavano le lettere greche; in non piccola quantità furono trasferiti a Roma presso il cardinale Bessarione, e poi da lì a Venezia; quanti erano scampati a tale dispersione furono distrutti dai Turchi, che saccheggiarono il monastero"'.

Il medico e umanista pugliese Antonio de Ferrariis, alias il Galateo, ritrae in questa breve descrizione la sua presunta visita al monastero idruntino prima della sua distruzione, nel 148o, avvenuta in seguito alle scorrerie turche. Il cenobio, fondato nel 1088 da Boemondo, principe di Taranto e di Antiochia, giusto nel crocevia tra Costantinopoli e Roma, divenne tra l'XI e il XV secolo un crogiuolo intellettuale formato da monaci eruditi, letterati, poeti e scienziati dedicati alla conservazione e alla diffusione del patrimonio storico e letterario. Questo monastero tuttavia, come anche i resti archeologici evidenziano, venne eretto su una struttura preesistente, probabilmente dell'epoca di Leone III l'Isaurico, dove i monaci trovarono rifugio dalle invasioni persiane e dalla persecuzione iconoclasta, divenendo pertanto l'epicentro a partire dal quale la regola basiliana si estese in tutto il meridione. Infatti, alle casupole precedenti, il monastero passò ad inglobare anche i feudi circostanti, vale a dire le

\footnotetext{
${ }^{1}$ A. DE FERRARIIS, De situ Japigiae, 8, 7-9 (ed. D. De Filippis, La Iapigia [Liber de situ Iapygiae], Galatina, 2005, p. 36-37): «Post hunc coenobium est divo Nicolao dicatum mille et quingentis passibus ab Hydrunto distans. Hic monachorum magnii Basilii turba convivebat. Ii omni veneratione digni, omnes litteris Graecis et plerique etiam Latinis instructi, optimum sui praebebant spectaculum. Quicunque Graecis litteris operam dare cupiebant, iis maxima parte victus, praeceptor, domicilium sine aliqua mercede donabatur. Sic res Graeca, quae quotidie retro labitur, sustentabatur. Fuit temporibus proavorum nostrorum, stante aula Constantinopolitana, vir philosophus Nicolaus Hydrontinus, cuius ante Turcarum transitum plures libri de logica et philosophia in hoc monasterio habebantur. Hic, abbas huius monasterii factus et Nicetas nominatus, saepe a summo Pontifice ad Imperatorem et ab illo ad summum Pontificem permeabat ad componendas res, quando inter Pontificem et Imperatorem aliqua contentio aut de orthodoxa fide aut de alia re oriebatur. Erat enim hic vir gravissimae auctoritatis et sanctissimorum morum, ut qui de philosophia ad religionem commigraverat. Hic sumptui minime parcens, quos per universam Graeciam invenire potuit librorum omnis generis bibliothecam in hoc coenobio congessit. Quorum magna pars negligentia Latinorum et contemptu litterarum Graecarum periit; non parva pars Romam ad Bessarionem cardinalem deportata est et inde Venetias; partem quae superfuerat Turcarum, qui monasterium populati sunt, bella absumpserunt».
} 

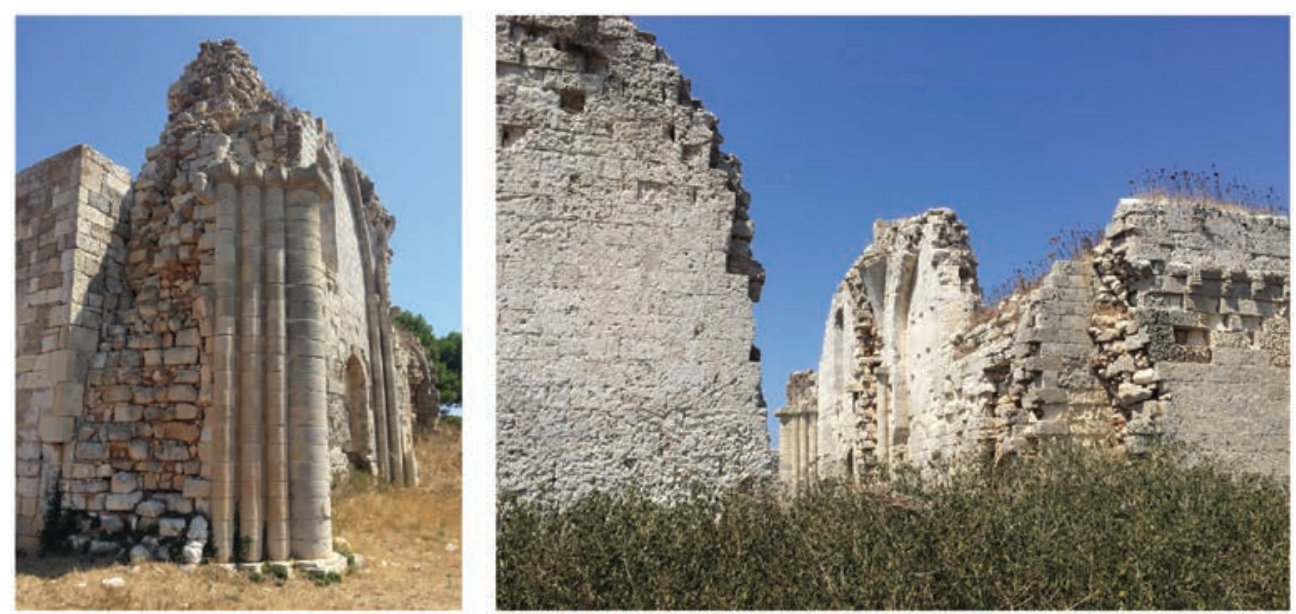

di opere teologiche, filosofiche e letterarie. Il Gregorovius, a questo proposito, affermava che "la biblioteca claustrale di San Nicola presso Otranto contava tra le più antiche dell'Occidente, ed era forse per origine anche anteriore a quella fondata da Cassiodoro nel cenobio Vivariense. V'erano manoscritti greci in gran copia. Il cardinale Bessarione ne fece sua una parte, la quale poi a Venezia, insieme con tutta la biblioteca di lui, andò miserabilmente perduta. I manoscritti rimasti ancora nel chiostro, li distrussero più tardi i Turchi, quando nel 1480 s'impadronirono di

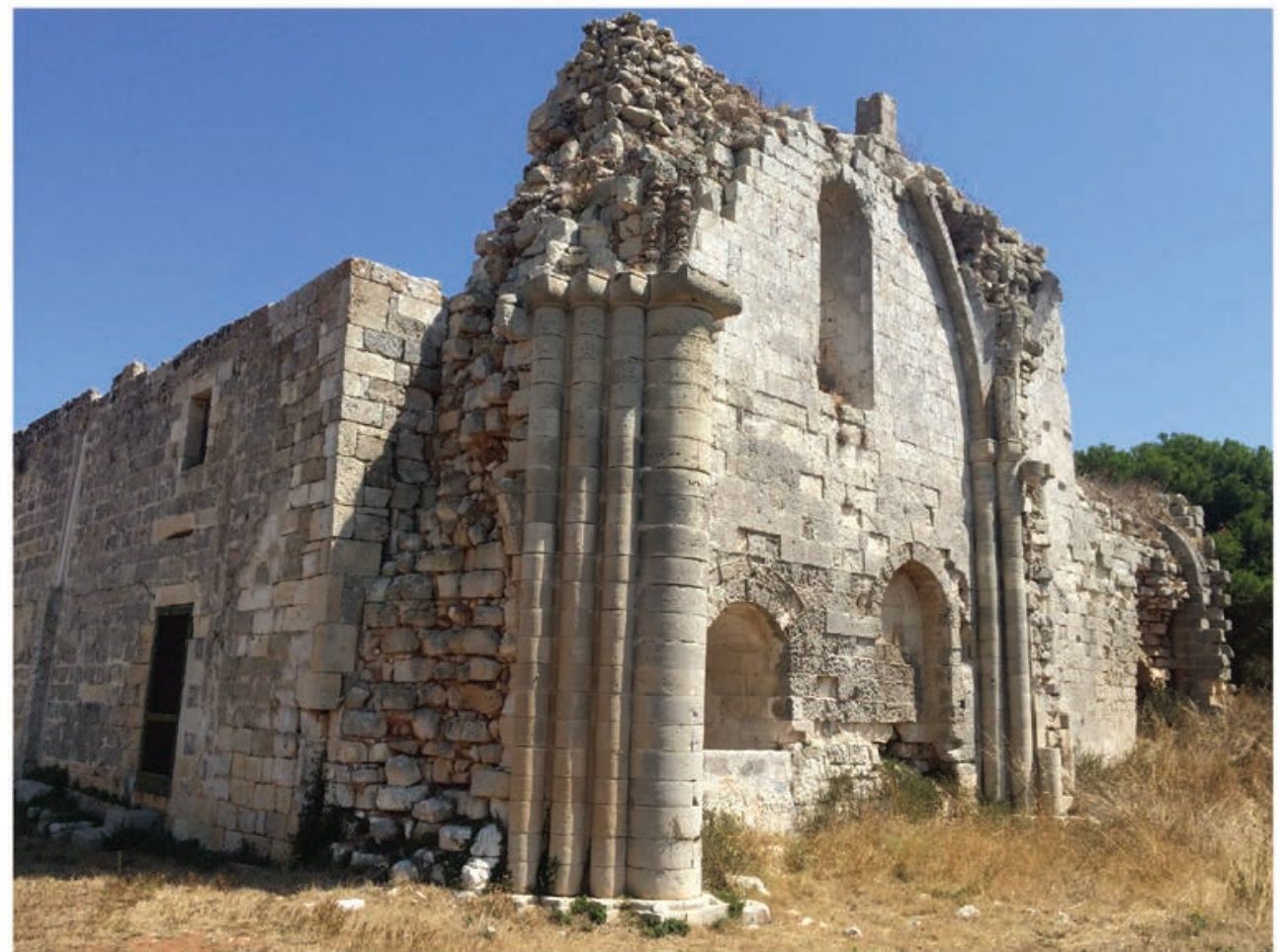
Otranto. Galateo parla del fatto nel suo libro De situ Japygiae. Egli stesso era riuscito a porre in salvo un codice greco, del quale fece presente al papa Giulio II: peccato che il manoscritto, oltre la donazione di Costantino, non contenga nulla di più importante!"3.

\section{STORIA DEL CODICE}

Questa testimonianza, sebbene aleatoria, costituisce la seconda notizia sull'abbazia posteriore a quella di de Ferrariis, la quale riassume, a grandi linee, gli argomenti proposti in questa comunicazione, ovvero la ricostruzione dell'attività dello scrittorio e della biblioteca a partire dall'unica fonte, un manoscritto della Biblioteca Reale dell'università di Torino ${ }^{4}$, conservato sotto la segnatura Graecus 216 (ex Codex Taurinensis Graecus C III 17). La maggior parte di questo testo è costituita da un Fig. 1. Resti delle colonne e la masseria annessa (foto Miguel Ángel Segura Monje). typikon, un documento relativo alle

metochie $^{2}$, le grangie e le laure, arrivando così a diventare uno dei cenobi con il latifondo più ampio del sud Italia.

Ed è proprio qui dove nasce lo scriptorium casulanum, una vera e propria officina di produzione testuale grazie al lavoro paziente dei calogeri basiliani, i quali dividevano le loro giornate tra l'ascesi, la mortificazione e la copiatura costituzioni alla base dell'abbazia, un formato letterario molto tipico nell'Italia meridionale, soprattutto nell'area bizantina, e seguace, nella sua struttura contenutistica, delle Regulae fusius tractatae del padre cappadoce Basilio e, come avremo modo di analizzare più avanti, delle Poenae monasteriales ${ }^{5}$ di Teodoro Studita.

${ }^{2}$ Le metochie, secondo la definizione di Daquino (C. DAQUINO, Bizantini in Terra d'Otranto. San Nicola di Casole, Lecce, 200o, p. 17), erano "le succursali di un monastero, ovvero case monastiche create dal cenobio principale, dal quale dipendevano direttamente, e quindi non avevano un proprio igumeno ma erano guidate da un monaco incaricato dall'igumeno". Per grangie e laure, invece, si intendono, rispettivamente, le fattorie e gli appezzamenti di terreno coltivati dai monaci a mo' di sussistenza.

${ }^{3}$ F. GREGOROVIUS, Apulische Landschaften, Leipzig, 1877 (trad. it. F. Mariano, Nelle Puglie, Firenze, 1882, rist. anast, Pianoro, 1975 p. 374 ).

${ }_{4}^{4}$ Tuttavia, a quanto pare, il Galateo non è stato l'unico a menzionare il monastero di San Nicola di Casole. Come citato da C. DAQUINO [op. cit. (n. 2), p. 17] e da Mazzotta (O. MAZZOTTA, Monaci e libri nel Salento medievale. Il monastero di San Nicola di Casole, in Sergio Stiso tra Umanesimo e Rinascimento in Terra d'Otranto, Galatina, 2012 p. 149-164: p. 149), lo storico Tafuri lesse brani di una Historia Monasterii S. Nicolai de Hydrunto, redatta dal monaco casolano Mauro di Otranto, e, a tutt'oggi, andata perduta.

${ }^{5}$ Sull'influenza dei typika bizantini in Italia meridionale, gli studi più rilevanti sono stati quello di T. MINISCI, I typica liturgica dell'Italia bizantina, in Bollettino della Badia greca di Grottaferrata $(=B B G G)$, 7 (1953), p. 97-104; quello di A. PERTUSI, Rapporti tra il monachesimo italo-greco ed il monachesimo bizantino nell'alto medioevo, in La chiesa greca in Italia dal VII al XVI secolo, 2, Padova, 1972, p. 473-520 (in particolar modo, p. 485-486), e quello di P. ROUGERIS, Ricerca bibliografica sui Typika italogreci, in BBGG, 27 (1973), p. 11-42. Sul ruolo preponderante della regola studita nello sviluppo del monachesimo bizantino in Oriente vedasi lo studio dettagliato di J. LEROY, La réforme studite, in Orientalia Christiana Analecta $(=$ OCA) 153, Roma, 1958, p. 181-214, e sempre dello stesso autore, la monografia Studistisches Mönchtum. Spiritualität und Lebensform, Graz, 1969. Per quanto concerne la penisola italica, in particolar modo nel settore meridionale, vd. T. MINISCI, Riflessi studitani nel monachesimo italo-greco, in Il monachesimo orientale, OCA, 153 [1958] p. 215-233). 
Prima della scoperta del testo, nella metà del XVIII seco$l^{6}$, le informazioni sul monastero erano per lo più di tipo cronachistico e non del tutto affidabili ${ }^{7}$; il Codex graecus fornisce, al contrario, sebbene non in maniera del tutto esaustiva, squarci della vita quotidiana di Kasoulon, come per esempio la sua struttura, la sua organizzazione interna, il suo scriptorium e la biblioteca. Nel cenobio la vita contemplativa, assieme al rigore proprio della regula, veniva alternata all'attività culturale la quale, mediante la copiatura dei manoscritti di autori e di opere di carattere prevalentemente sacro e, allo stesso tempo, di altri di impronta più secolare, permise a Casole e al suo laboratorio copistico di diventare un centro nevralgico di cultura e diffusione del sapere, sia nell'oriente bizantino sia nell'occidente cristiano.

\section{CARATTERISTICHE DEL CODICE}

La storia del Codex Taurinensis è decisamente vivace, tenendo in considerazione le vicissitudini e il suo periplo dal centro idruntino sino all'arrivo nella biblioteca dell'ateneo torinese. Il volume, sfuggito fortunamente al saccheggio turco del 1480 e al rogo della biblioteca regia del 1904, ne fu però seriamente compromesso, motivo per il quale fu riparato nella badia di Grottaferrata ${ }^{8}$, a causa degli ingenti danni subiti, oltre che dal fuoco, anche dall'uso grossolano di acqua. Fortunatamente però il manoscritto, per incarico del consigliere De Simone, era stato affidato, un decennio prima, al padre Giuseppe Cozza Luzi, il quale ne aveva realizzato una copia corredata di traduzione latina, attualmente conservata nella biblioteca provinciale di Lecce con la segnatura $\mathrm{ms} 201^{9}$. Oltre a questa trascrizione, ampie parti del testo furono riportate dall'Omont nel 189o, in un articolo pubblicato sulla Revue des études grecques ${ }^{10}$, le quali, a loro volta, rimandano ad alcune accennate anteriormente da Diehl nel suo articolo del $1886^{11}$.

Il codex, agli occhi dei tre succitati studiosi, si presentava originariamente come un volume dalle misure di 255 millimetri per 188, composto da $183 \mathrm{ff}$, oltre ad un foglietto preli- minare, scritto in un'elegante minuscola, la quale, seguendo i criteri paleografici, sarebbe rapportabile alla seconda metà del dodicesimo secolo ${ }^{12}$. Nel primo foglio, appare il nome

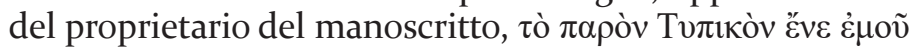

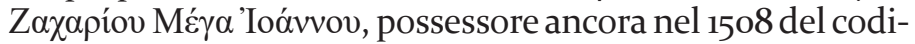
ce, un trentennio dopo la distruzione compiuta dai Turchi ${ }^{13}$. Il manoscritto, come dicevamo, era composto nella sua maggior parte da un typikon, a eccezione dei primi cinque folia, nei quali, oggetto della nostra analisi, recava una miscellanea di documenti di ogni tipo, tra i quali risaltavano in particolar modo le norme riferite allo scriptorium e alla sua biblioteca. Sotto la guida dell'igumeno, infatti, i monaci spendevano la maggior parte della giornata alla copiatura dei tomi più rari, arrivando persino a trascrivere testi come le opere di Omero, di Esiodo e di Aristofane. La struttura dello scriptorium, come il manoscritto evidenzia, era decisamente gerarchica: facendo capo all'igumeno, il bibliotecario, o meglio conosciuto come bibliofilace, organizzava il lavoro dei protocalligrafi, i quali, da parte loro, sorvegliavano il lavoro del resto degli amanuensi, evitando possibili distrazioni o errori grossolani dei copisti nella trascrizione dei testi, i quali, a loro volta, venivano puniti severamente.

Grazie all'articolo dell'Omont, che vide personalmente il documento prima che fosse danneggiato dall'incendio, ed alla trascrizione in greco, accompagnata a fronte da una traduzione in latino, a cura dell'abate di Grottaferrata Cozza-Luzi ${ }^{14}$, si può constatare, nella copia giunta ai nostri giorni, come invece, dopo il restauro di Grottaferrata, la posizione degli stessi, in seguito alla riparazione, fosse stata cambiata dall'originaria posizione 3,4 e 5 alla collocazione in un unico foglio, quello sopravvissuto all'incendio, vale a dire il 180 recto $^{15}$. Tuttavia, questa nuova foliazione non permette la ricostruzione delle norme di Casole, suddivise in capitoli, alle quali Pasini faceva riferimento, ossia i capitoli 21-56. Infatti, mentre i capitoli $44-56$ vengono identificati con il f. 180 (f. 5, prima dell'incendio), non rimane documento alcuno dei capitoli 21-43 (che dovrebbero corrispondere al folio 179, vecchio f. 4 , del quale si è persa ogni traccia) ${ }^{16}$.

\footnotetext{
${ }^{6}$ Il manoscritto, a parere dell'Omont [H. OMONT, Le typicon de S. Nicolas de Casole, près d'Otrante, in Révue des études grecques $(=$ REG), III (1890) p. 381], fu descritto per la prima volta dal Pasini, nella sua opera Codices mss. bibliothecae regii Taurinensis athenaei, nel 1749 e segnalato, per il suo grande valore documentale, da Ermanno Aar all'interno di una recensione pubblicata nell'Archivio storico italiano (4ª serie, tomo VI, [188o], p. 318-319). Successivamente scaturì, a partire da questa segnalazione, lo studio di Diehl e quello dell'Omont.

${ }^{7}$ H. HOUBEN, La conquista turca di Otranto (1480): il problema delle fonti salentine, in H. Houben (a cura di), La conquista turca di Otranto (1480) tra storia e mito, Atti del Convegno internazionale di studio (Otranto - Muro Leccese, 28-31 marzo 2007), 2, Galatina, 2008, p. 5-20.

${ }^{8}$ N. BORGIA, Un codice greco ricuperato, in Accademie e biblioteche d'Italia, XIX (1939), p. 97-102.

9 O. MAZZOTTA, op. cit., p. 150; G. COZZA-LUZI, Lettere Casulane, Reggio Calabria, 1900; M. MUCI, Guida al carteggio di L.G. de Simone, con le Lettere casulane di G. Cozza-Luzi, Lecce, 2006 (Quaderni della Biblioteca "N. Bernardini”, 7).

${ }^{10} \mathrm{H}$. OMONT, Le typicon de S. Nicolas de Casole, près d'Otrante, in REG, III (1890), p. 381-391.

${ }^{"}$ C. DIEHL, Le monastère de S. Nicolas di Casole près d'Otrante, d'après un manuscrit inédit, in Mélanges d'archeologie et d'histoire, 6 (1886), p. 173-188.

${ }^{12}$ La descrizione del manoscritto è del G. COZZA-LUZI, op. cit. (n. 9), n. 2, p. 6 [vd. M. MUCI, op. cit. (n. 9), p. 84]. Sul ductus e i criteri paleografici per la datazione, $v d$. D. ARNESANO, Gli epitimia di Teodoro Studita. Due fogli ritrovati del dossier di Casole, in Byzantion, (80), 2010, p. 9-37.

${ }^{13}$ In base a quanto sostenuto da Arnesano, in un articolo ricco di dettagli [D. ARNESANO, op. cit. (n. 12), p. 18], più che trattarsi del codice completo, la nota riferita a Zaccaria farebbe riferimento unicamente al f. 4, separato nel 1508 dal Codex taurinensis e tra le mani del Megagianni, secondo la trascrizione di Omont [H. OMONT, op. cit. (n. 10), p. 382] o del figlio del maestro Giovanni, secondo G. COZZA-LUZI, op. cit. (n. 9), p. 8; M. MUCI, op. cit. (n. 9), p. 85. ${ }^{14} \mathrm{La}$ copia in questione si trova attualmente nella biblioteca della provincia di Lecce, sotto la segnatura Ms 201. Il valore di questa copia in latino è di grande importanza, giacché, per quanto riguarda le parti distrutte in seguito all'incendio, queste possono essere ricostruite attraverso di essa [G. COZZA LUZI, Lettere Casulane, Reggio Calabria, 1900; N. BORGIA, op. cit. (n. 8), p. 97-102; H. OMONT, op. cit. (n. 10), p. 381-391].

${ }_{15}$ Giuseppe Pasini, tra i primi a redigere una descrizione del codice torinese (G. PASINI, Codices manuscripti Bibliothecae Regii Taurinensis Athenaei, I, Torino 1749, p. 308-309), si accorse della presenza, nei fogli 4-5, di testi composti da regole, suddivise in 56 capitoli. Egli, tuttavia, non ravvisò, come vedremo più avanti, l'archetipo di Stoudion, ma sostenne, bensì, che queste erano norme proprie del monastero ("sequuntur regulae, ni fallor, ejusdem Monasterii capitibus quinquaginta sex comprehensae, quarum priorum viginti desiderantur", ibid., p. 309) a differenza di Cozza-Luzi, che ne vide chiaramente l'origine studitana [G. COZZA-LUZI, op. cit. (n. 9), p. 8-9; M. MUCI, op. cit. (n. 9), p. 85].

${ }^{16}$ Sulla spinosa questione della foliotazione, vedasi in dettaglio D. ARNESANO, op. cit. (n. 4), p. 14, n. 4.
} 

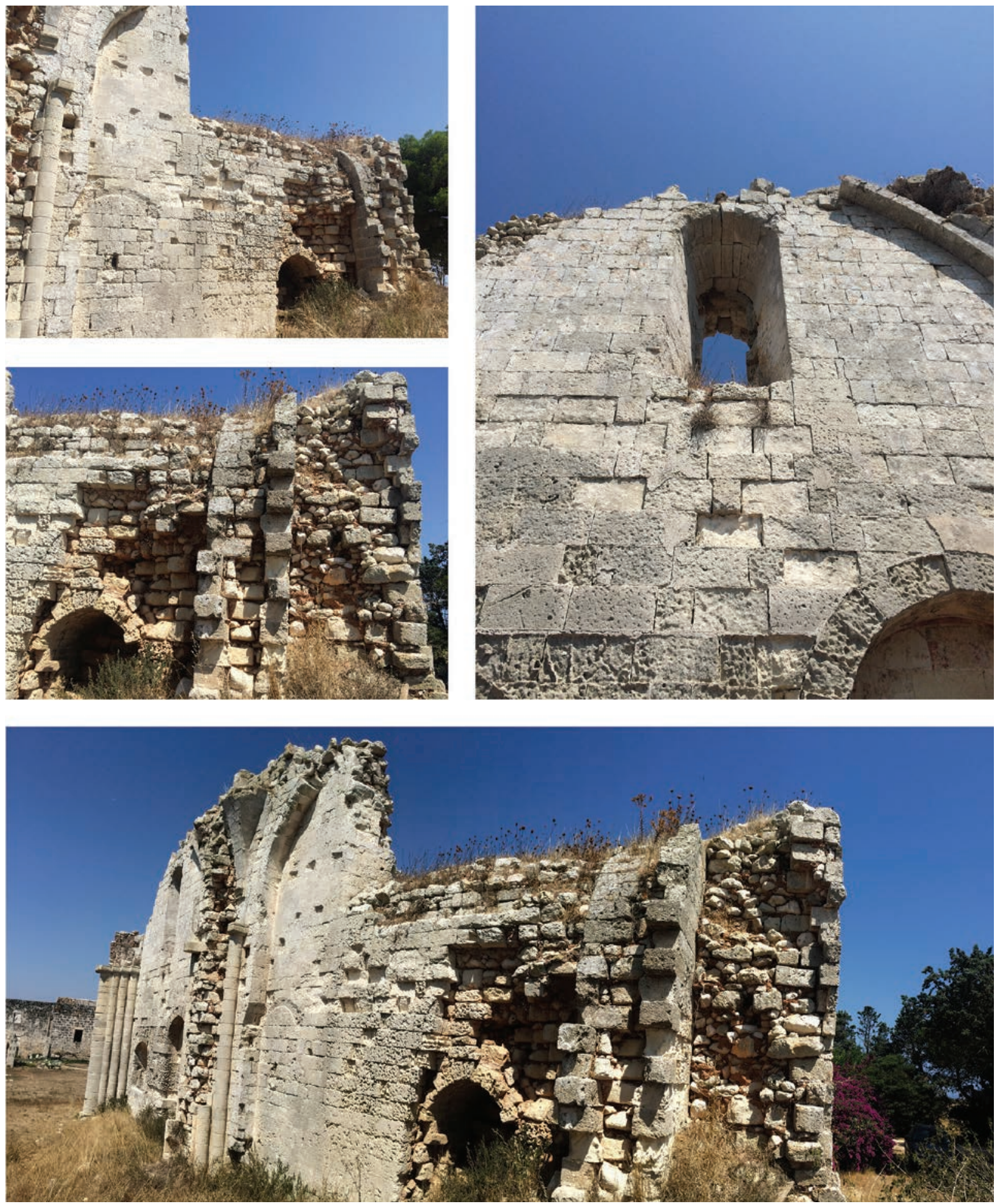

Fig. 2. Dettagli del cenobio (foto Miguel Ángel Segura Monje)

Grazie alla trascrizione effettuata nel 1890 da Omont, questi, che contenevano le norme alla base dello scriptorium basiliano ${ }^{17}$ possono essere letti, tuttavia, in parte. Le prescrizioni, come possiamo confrontare mediante la tavola sottostante, non sono nient'altro che una riproduzione di quelle del monastero di Stoudion, riprodotte nel volume 99

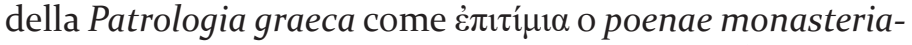
$l s^{18}$. Risulta interessante, mediante questo confronto tra $\mathrm{i}$ due testi, poter ricostruire, grazie alle ulteriori istruzioni presenti nel testo degli غ̇ंıtíuı di Teodoro Studita, come funzionasse il centro copistico. In primo luogo, per quanto riguarda la scuola calligrafica, vigeva una rigorosissima disciplina. Non era permesso agli amanuensi commettere il minimo errore ed era loro d'obbligo trattare con cura gli strumenti forniti dallo scrittorio:

${ }_{17}$ H. OMONT, op. cit. (n. 10), p. 391. L'autore, eppure, come sottolinea Arnesano [D. ARNESANO, op. cit. (n. 4), p. 15] non ne ravvisó l'origine studitana,

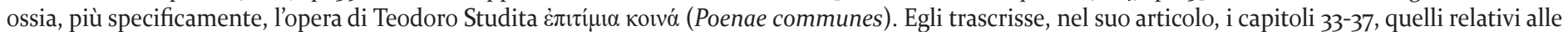
pene previste per gli scribi negligenti, i quali sono da ricondurre al f. 4 .

${ }^{18}$ Patrologia graeca, 99, col. 47-49; 53-6o. Vedasi altresì la nota, a questo proposito, di Featherstone e Holland (J. FEATHERSTONE, M. HOLLAND, A note on penances prescribed for negligent scribes and librarians in the monastery of Studios", in Scriptorium, 36 [1982], p. 258-260). 


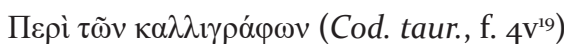

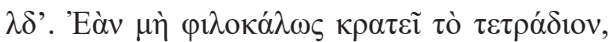

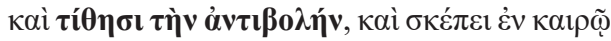

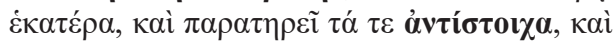

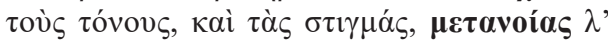
кaì $\rho$ '.

"Se non tratta con cura il quaternione ${ }^{20}$, e non fa la collazione e non controlla allo stesso tempo entrambi, e non fa attenzione agli aspetti ortografici corrispondenti ${ }^{21}$, agli accenti e alla punteggiatura, [riceva come castigo] 130 metanie $^{22}$ )".

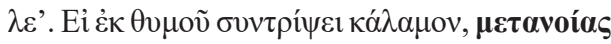
$\lambda$,

"Se, per un attacco di collera, spezza la penna, 30 metanie".

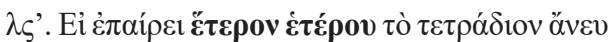

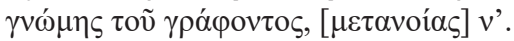

"Se prende un quaternione di un altro senza il consentimento di chi lo sta copiando, 50 metanie".

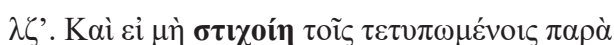

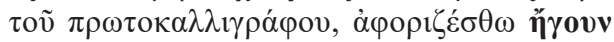

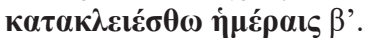

"Se non dovesse seguire i canoni stabiliti dal protocalligrafo, sia scomunicato ${ }^{23}$ e rinchiuso per due giorni".

Anche i dettami relativi all'uso dei testi nella biblioteca seguivano dei parametri altrettanto rigidi:

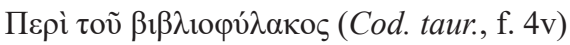

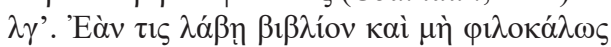

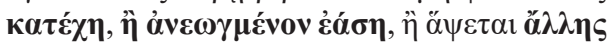

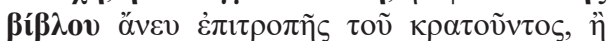

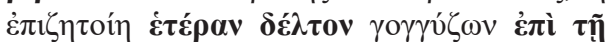

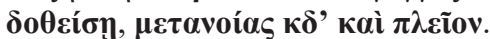

"Qualora qualcuno avesse preso un libro e non lo avesse tenuto con cura, o lo lasciasse aperto, o prenderà un altro libro senza il permesso del proprietario, o nel richiedere un altro libro, borbotterà contro chi glielo ha dato [riceva come castigo], 24 metanie e più"24.

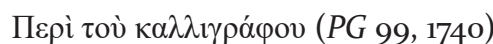

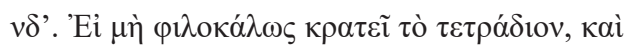

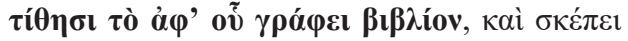

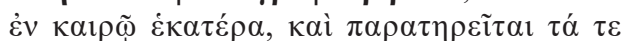

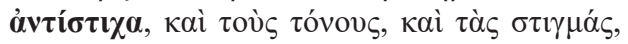

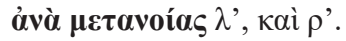

"Se non tratta con cura il quaternione, e non mette a posto il libro di colui che lo copia, e non controlla allo stesso tempo entrambi, e non fa attenzione agli aspetti ortografici corrispondenti, agli accenti e alla punteggiatura, [riceva come castigo] 130 metanie)".

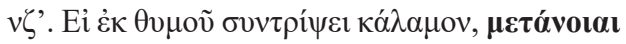
$\lambda$.

"Se, per un attacco di collera, spezza la penna, 30 metanie".

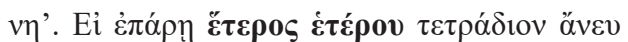

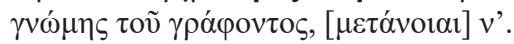

"Se prende qualcuno un quaternione di un altro senza il consentimento di chi lo sta copiando, 50 metanie".

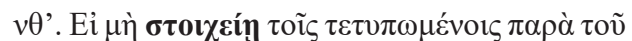

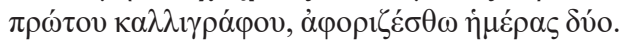

"Se non dovesse seguire i canoni stabiliti dal protocalligrafo, sia scomunicato per due giorni”.

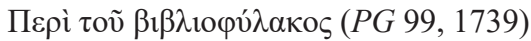

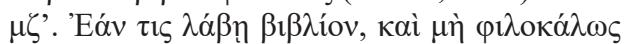

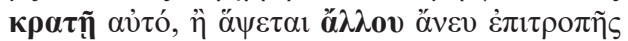

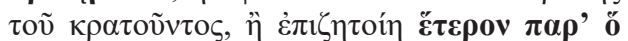

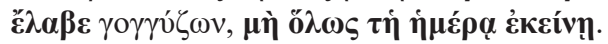

"Qualora qualcuno avesse preso un libro e non lo avesse tenuto con cura, o dovesse prendere un altro libro senza il permesso di colui che lo ha in possesso, o cercasse un altro libro, borbottando contro chi glielo ha dato, non gli verrà concesso in nessun modo durante lo stesso giorno".

\footnotetext{
${ }^{19}$ Per la numerazione dei folia, $v$ d. D. ARNESANO, op. cit. (n. 4), p. 28, che riporta un nuovo testo critico e una tavola (la n. 4), che riporta, a sua volta, la foto del folio del manoscritto.

${ }^{20}$ Il quaternio è un fascicolo composto da 4 fogli dal doppio formato (8 carte, 16 pagine).

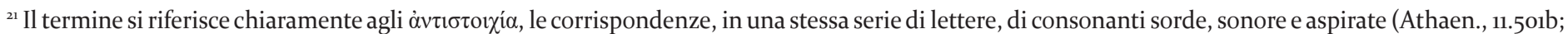

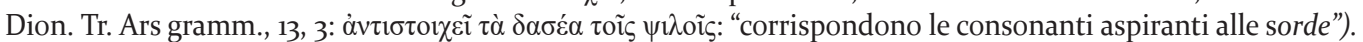

${ }^{22}$ Per metania si intende l'atto della prostrazione, nella maggior parte manifestato in forma di genuflessione, per dimostrare umiliazione o pentimento (vd. A. VACCARO, Dizionario dei termini liturgici bizantini e dell'Oriente cristiano, Lecce, 2010, p. 220-221; J. LEROY, op. cit. [n. 4], p. 210-211).

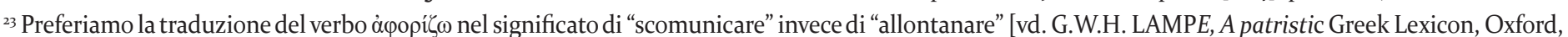
2014, p. 279; A. VACCARO, op. cit. (n. 22), p. 75] dal momento che, come l'epitimion di Casole ben spiega, il castigo della scomunica era accompagnato dalla reclusione, ossia l'abbandono delle pratiche quotidiane della vita cenobitica e l'assoluto isolamento per la riflessione (J. LEROY, op. cit. [n. 4], p. 210-211. Per il concetto di “isolamento", vd. Lampe, op. cit., p. 279: "put away in monastery prison").

${ }^{24}$ Il paleografo Arnesano, nel suo articolo (D. ARNESANO, op. cit. [n. 4], p. 9-37) sottolinea l'influenza del typikon di Teodoro Studita nell'ambito cenobitico italo-greco, soprattutto in riferimento alle somiglianze nelle parti relative alle pene inflitte nel monastero (p. 15).
} 
A differenza di queste brevi note presenti nel Codex, le costituzioni studitane entrano molto più nel dettaglio circa $i$ doveri del $\beta \imath \beta \lambda$ ıо v́ $\lambda \alpha \xi$ il quale, doveva controllare che il tesoro del cenobio, i suoi preziosi volumi, ritornassero al proprio posto, dopo l'uso, e a un determinato orario: "prim'ancora che suoni il simandro vespertino, colui che è incaricato dei libri lo fa suonare ancora una volta, e tutti, in ordine secondo

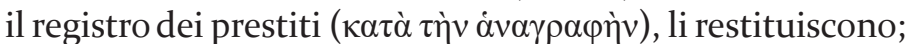
se qualcuno si intrattiene nella restituzione del libro ( $\varepsilon i \grave{\delta} \delta$

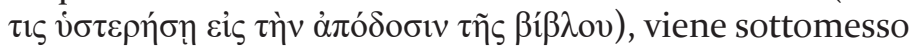

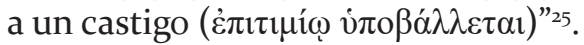

Poteva succedere infatti che, in determinate occasioni, qualcuno tra i monaci fosse leggermente indisciplinato e preferisse tenere il libro con sé nella sua cella invece di restituirlo. Questo era considerato, nel regolamento studitano, un atteggiamento riprovevole, e come tale, meritava un severo castigo:

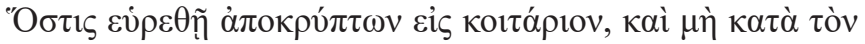

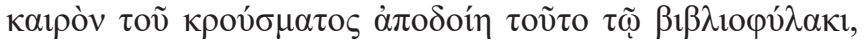

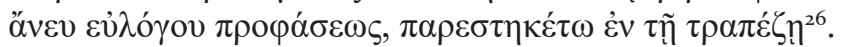

"Chiunque venga sorpreso nascondendo un libro nella sua cella, o no lo restituisca al bibliotecario nel momento del tocco (del simandro) senza una scusa valida, rimanga in piedi nel refettorio".

Il bibliotecario non solo doveva far attenzione a che i libri venissero restituiti nel momento dovuto, ma anche che il loro stato di conservazione fosse ineccepibile:

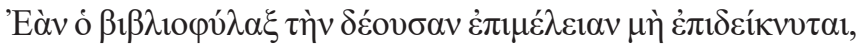

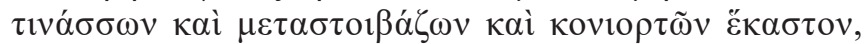
$\xi \eta \rho о \varphi \alpha \gamma \varepsilon i \tau 0^{27}$.

"Se il bibliotecario non mostra la cura necessaria, bistrattando ogni volume, impilandolo e facendogli accumulare polvere, sia messo a dieta asciutta".

Tuttavia, nonostante le informazioni forniteci dal Codex, possiamo, ricorrendo però alle informazioni delle costituzioni studitane, ricostruire il lavoro quotidiano degli amanuensi. Il protocalligrafo doveva distribuire, in maniera imparziale $(\dot{\varepsilon} \mu \pi \alpha \theta \tilde{\omega} \varsigma)$, i compiti dei copisti ( $\tau \grave{\alpha} \dot{\varepsilon} \rho \gamma o ́ \chi \varepsilon 1 \rho \alpha)$, prestando particolare attenzione alle pergamene ( $\alpha i$ $\mu \varepsilon \mu \beta \rho \alpha ́ v \alpha 1)$ e facendo attenzione che gli strumenti destinati

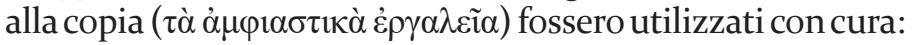

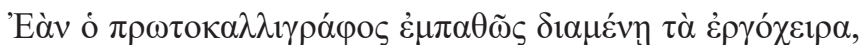

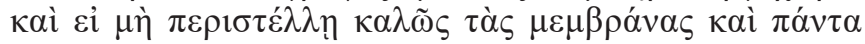

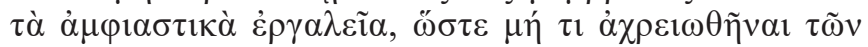

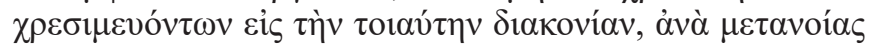

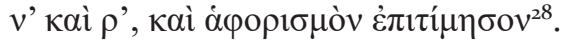

"Nel caso in cui il protocalligrafo non dovesse distribuire in maniera imparziale i compiti manuali, e non trattare con attenzione i pergamini e tutti gli strumenti destinati alla copia, e, di conseguenza, tutti gli strumenti destinati a tale servizio risultino inappropriati, venga castigato con 150 metanie e rinchiuso".

La responsabilità del protocalligrafo era onerosa pertanto, in particolar modo per quello che riguardava la qualità della copia e il controllo scrupoloso del lavoro dell'amanuense nella riproduzione dei codici. Molti scribi infatti, trascrivendo i testi, non si limitavano unicamente alla loro copia, ma azzardavano persino un'interpretazione personale:

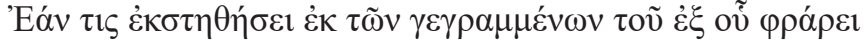

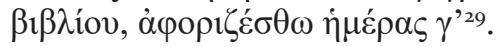

"Se qualcuno si discosterà, nel libro che copia, da quello che è riportato nel testo, sia scomunicato per 3 giorni".

La pena per questo tipo d'errore, voluto intenzionalmente, non si limitava solo all'allontanamento, ma anche una punizione di tipo corporale:

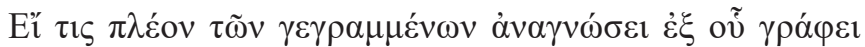
$\beta 1 \beta \lambda$ íov, $\xi \eta \rho о \varphi \alpha \gamma \varepsilon i \tilde{\tau} 0^{30}$.

"Se qualcuno adotterà una lezione diversa da ciò che è riportato nel testo, che venga messo a dieta asciutta”.

Subito dopo la sezione dedicata alle norme che regolavano il laboratorio copistico dello scriptorium casulano, appariva, nei folia 181 recto e 182 verso la lista dei prestiti dei volumi conservati nella biblioteca. Circa il metodo di cessione, questo veniva realizzato mediante la consegna di una cedola, alla presenza di due monaci, incaricati del servizio di prestito ${ }^{31}$. Questa ipotesi, infatti, può essere estrapolata da un frammento presente nella lista dei prestiti, alla fine del folio 181 recto:

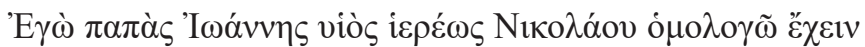

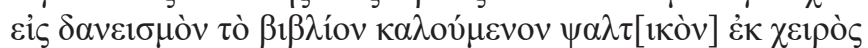

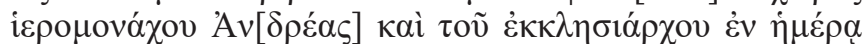

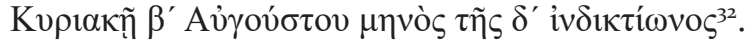

\footnotetext{
${ }^{25}$ Patrologia graeca, 99, col. 6o.

${ }^{26}$ Patrologia graeca, 99, col. 49. Sulla traduzione di $\tau \rho \alpha ́ \pi \varepsilon \zeta \alpha$ come refettorio, $v d$. A. VACCARO, op. cit. (n. 22), p. 304. La punizione in base alla quale il monaco castigato doveva rimanere in piedi durante la refezione ( $\pi \alpha \rho \alpha \sigma \tau \alpha \dot{\sigma} \mu \rho \varsigma)$, era frequente nei monasteri (A.M. TALBOT, Mealtime in monasteries: the culture of the Byzantine refectory, in L. Brubaker, K. Linardou (ed.), Eat, drink and be merry (Luke 12:19). Food and wine in Byzantium. Papers of the 37th Annual Spring symposium of Byzantine studies, Aldershot, 2007, p. 109-126.

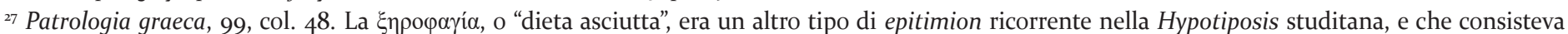
essenzialmente nel proibire al castigato di ingerire alimento liquido e permettergli unicamente l'assunzione di quello secco, asciutto ( $\xi \eta \rho o ́ \varsigma)$. Questo tipo di mortificazione, in ogni modo, era praticata durante il periodo quaresimale, e consisteva essenzialmente nel nutrirsi di pane, acqua e legumi secchi ( $v d$. J. LEROY, op. cit. [n. 4], p. 210-211; A. VACCARO, op. cit. [n. 22], p. 320).

${ }^{28}$ Patrologia graeca, 99, col. 60.

${ }^{29}$ Patrologia graeca, 99, col. 55 .

${ }^{30}$ Patrologia graeca, 99, col. 56 .

${ }^{31}$ Anche lo storico salentino, seguendo la tesi di Charles DIEHL, op. cit. (n. 11), p. 187 conferma la stessa ipotesi (C. DAQUINO, op. cit. [n. 2], p. 134).

${ }^{32}$ L'edizione del testo utilizzata è quella di A. APOSTOLIDIS, Il typikon di S. Nicola di Casole secondo il codice Taur. Gr. C III 17, Introduzione, testo critico, indici, Roma-Bari, 1983-1984, p. 68.
} 

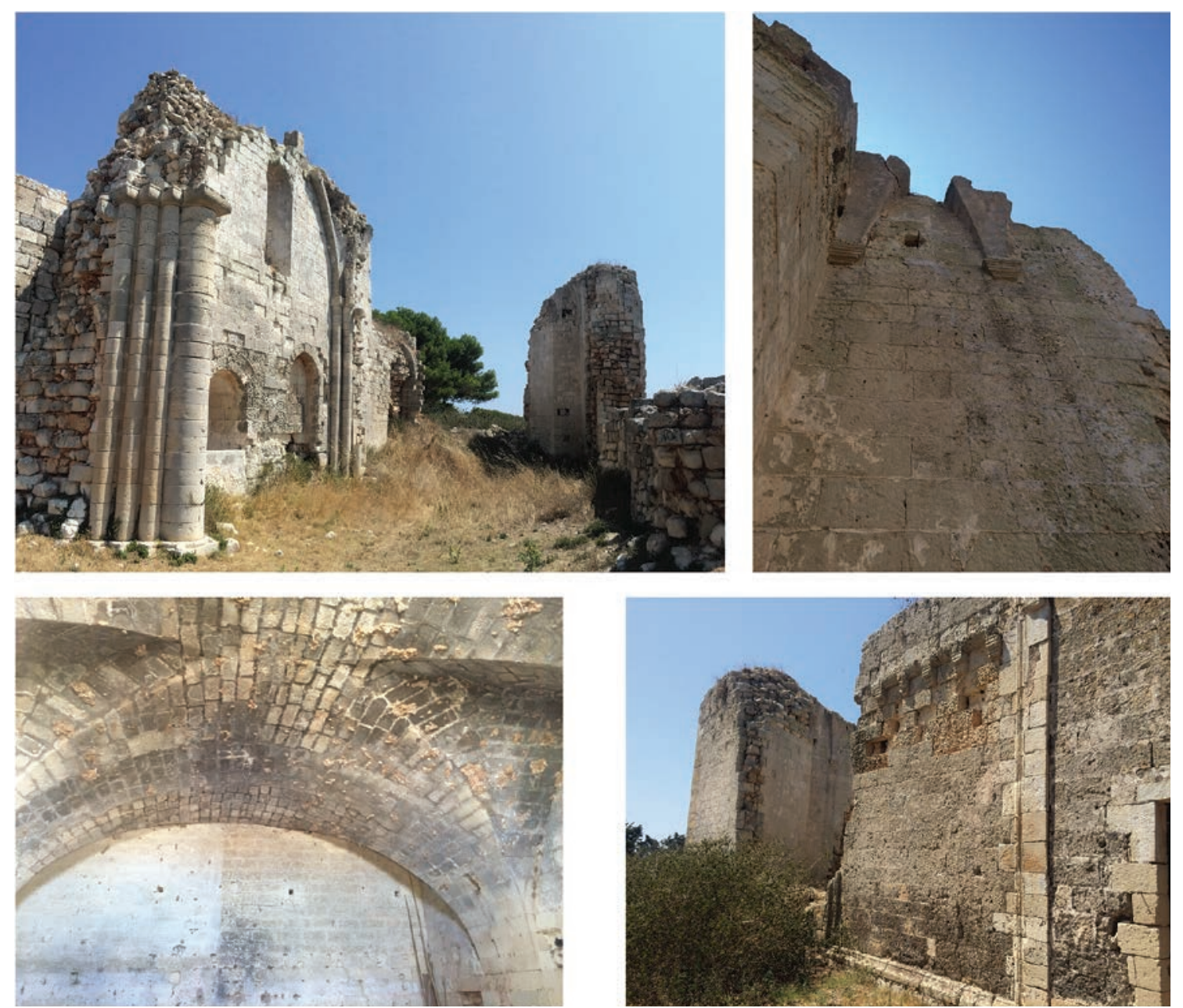

Fig. 3. Foto panoramica e mura perimetrali della masseria (foto Miguel Ángel Segura Monje).

"Io papa Giovanni, figlio del sacerdote Nicola, dichiaro di aver ricevuto in prestito un libro chiamato Psalt(icón) [= Salterio] dalle mani del ieromonaco e dall'(ieromonaco) ecclesiarca, nel giorno di domenica 2, del mese di agosto, dell'indizione 4 ".

L'ipotesi in base alla quale ci fossero, nella biblioteca, due autorità incaricate di garantire il prestito a chiunque fosse interessato a un volume è stata confermata anche da Diehl, il quale, a questo proposito, commentava: "un prêtre nommé Richard reconnait avoir reçu un livre de la main du hiéromonachos et ecclésiarque Sabas, en présence du hiéromonachos Jean" ${ }^{33}$. Il riferimento dello storico francese ci permette di confermare la nostra ipotesi, in base al quale la ricevuta di qualsiasi prestito, come quelle presenti nel manoscritto, erano firmate e registrate alla presenza di due ecclesiastici.

\section{PRESTITI}

A partire dalla traduzione da noi effettuata della lista dei prestiti presente nel codice, si possono rintracciare quindi i vari destinatari degli stessi, oltre a ravvisare, in alcuni casi per ipotesi, la loro localizzazione geografica, il tipo di volume presente nella biblioteca e gli utenti della stessa, i quali erano sia religiosi che laici. La diffusione esterna dell'attività bibliotecaria conventuale dimostra, in questo documento, come fosse decisamente stretta l'interrelazione tra mondo monastico e secolare, e come questo, da parte sua, usufruisse dei mezzi casulani per poter a sua volta produrre cultura. Altro dato notevole è la distribuzione geografica dei prestiti, i cui confini, a parte rare eccezioni, come il riferimento a Brindisi, coincide con i confini del latifondo monasteriale.

In totale, si possono, nella lista citata dal Codex, enumerare 68 prestiti, dei quali la maggior parte destinata a canonici e a tre laici, i quali, come si può leggere tra le righe del testo, sono due notai (il notaio Demetrio e il notaio Michele di Pietro) e un presunto giudice (non menzionato). Un altro fattore da tenere in considerazione è quello relativo ai dati identificabili col nome e lo status sociale, o, allo stesso tempo, a entrambi. Per quanto concerne l'aspetto sociale, la parte che è più rimarcata è quella che riporta i dati relativi alla professione. Spiccano su tutti, in particolar modo, i sacerdoti, che sommano in totale 39 prestiti; seguono i diaconi con 5; i monaci con 2, uno dei quali un egumeno di un monastero di una località sconosciuta; un presbitero, un lettore e una chiesa sotto il nome del capitolo; tra i laici, 2 notai e 1 giudice. Cinque lettori vengono inoltre identificati attraverso $\mathrm{i}$ padri, due dei quali sono sacerdoti e uno è probabilmente un giudice; degli altri due non viene menzionata la professione. Dei restanti 3 si riesce a leggere appena il nome.

Da un'attenta analisi dei prestiti rispetto alle opere, prevalgono i seguenti risultati: 20 vangeli, 7 eucologi, 5 triodi, 4 salteri e 4 profezie, 2 octoechi, 2 tipika e un esemplare di ciascuna delle seguenti opere: le commedie di Aristofane, gli elenchi sofistici di Aristotele, le omelie di Teofane il

${ }_{33}$ C. DIEHL, op. cit. (n. 11), p. 187. 
Cerameo, raggruppate nel volume Ceramita, gli Erotemata, un Lapidario, un Lessico, un Libro dei sogni, una Liturgia di San Basilio, un Messale, un Nomocanone, un Organon di Aristotele, un Praxapostolo, un Sinassario e uno Sticherarium menologii.

È evidente da questi rilievi che l'attività di prestito esterno della biblioteca casulana, eminentemente rivolta ai religiosi operanti nell'ambito geografico d'influenza politica ed economica del monastero, era di fatto finalizzata alla dotazione delle comunità religiose soggette, attraverso la copia, dei libri necessari alla liturgia e all'edificazione religiosa. Non mancano tuttavia le eccezioni: opere classiche vengono prestate a laici e due libri didattici a un prete e a un figlio di un prete. Si tratta delle commedie di Aristofane e degli Elenchi sofistici di Aristotele, prestati al notaio Michele di Pietro; del Libro dei sogni e del Lapidario, prestati a un non meglio identificato giudice; dell'Organon di Aristotele prestato a un tale Giovanni Tarentino; di un Lessico prestato al sacerdote Vitale di Castro e di una grammatica greca, gli Erotemata, prestata a Giovanni, figlio del sacerdote Vitalio di Marittima. Se a queste opere si aggiungono il Nomocanone prestato al sacerdote Andrea di Vignacastrisi e il Ceramita prestato al sacerdote Riccardo di Sanarica, abbiamo un campione di opere sufficientemente rappresentativo degli interessi culturali e professionali della società otrantina del XIII secolo.

Il quadro dei testi di erudizione scientifica e letteraria circolanti a Casole può essere arricchito inoltre dai riferimenti contenuti nei manoscritti di origine casulana, vale a dire dal Romanzo di Alessandro dello pseudo-Callistene e dalle Favole di Esopo contenuti nel Paris. gr. 1685. Oltre a questi, meritano anche una particolare menzione l'Iliade di Omero, le Omelie del Nisseno e del Damasceno, i Versi aurei di Pitagora e i Versi di un non meglio identificato Andrea Grasso, tutti frammenti miscellanei contenuti nell'Ambros. B 39 Sup. Non vanno inoltre dimenticati i Posthomerica di Quinto di Smirne e il Ratto di Elena di Colluto, delle cui copie casulane, andate disperse, si impossessò il cardinale Bessarione; né la Cronaca di Giorgio Cedreno, annotata più volte a Casole a fini didattici nel Vat. gr. 1903, né infine le Donazioni fatte dagli imperatori ai pontefici, opera contenuta nel codice donato a Giulio II dal Galateo, che lo dichiara proveniente da Casole, e la ricca biblioteca dell'umanista salentino Sergio Stiso, proveniente anch'essa in gran parte da Casole $^{34}$. Ė possibile, pertanto, trarre altre indicazioni di letture e prestiti a Casole da corrispondenze, cronache e cenni all'interno di altri manoscritti dello scriptorium idruntino.

\section{L'ELENCO DEI PRESTITI}

Risulta alquanto interessante analizzare alcuni frammenti del manoscritto ${ }^{35}$, puntualizzando, in determinati casi, le traduzioni precedent $\mathrm{i}^{36}$, per poter così localizzare geograficamente i toponimi utilizzati, chiarirne i dubbi e proporre delle nuove ipotesi.

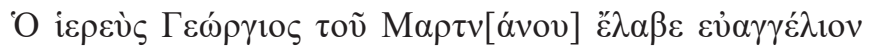

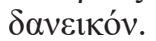

Si tratta del primo prestito, che si riscontra tanto nella trascrizione dell'Omont come in quella del Cozza Luzi, ma che è assente nel Codex. La traduzione di Gianfreda: "il sacerdote Giorgio (del martire Biagio) ha preso in prestito un Evangelio" risulta, a prima vista, leggermente approssimativa. Il nostro suggerimento prende in considerazione, al contrario, un fattore molto importante, quello geografico: "il

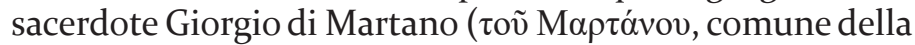
Grecia salentina, attuale Martano, non distante da Otranto) ha preso in prestito un volume dei vangeli". Riteniamo quindi, mediante questa ipotesi, che il prestito si riferisse a un sacerdote di una cittadina vicina al cenobio e non un riferimento al martire Biagio, come annota il Gianfreda.

Sempre a proposito di questa apparente difficoltà a localizzare geograficamente il luogo al quale si fa riferimento nella nota manoscritta, proporremo un altro esempio:

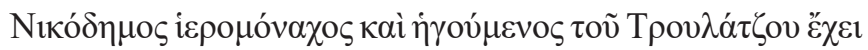

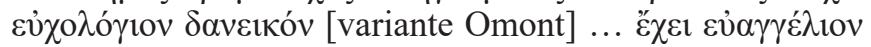

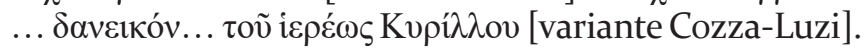

"Nicodemo, ieromonaco ed egumeno di Trulazzo, prende in prestito un eucologio [Omont], prende in prestito un vangelo [Cozza-Luzi] dal sacerdote Cirillo...”

In questo caso, le due varianti attestano il prestito di due testi distinti, un eucologio e un vangelo. Tuttavia, ambedue volumi hanno in comune un unico problema: la geo-localizzazione di Trulatzo, o come traduce Gianfreda, Turlazzo, giacché, prestando fede al testo, questo cenobio era governato da un igumeno, e, come tale, faceva capo a un monastero. Sfortunatamente però, non si è riusciti, e non si riesce tuttora, a causa della scarsezza delle fonti in merito, a conferire una localizzazione geografica precisa a questo toponimo, anche se Pasanisi fornisce un'indicazione molto convincente ${ }^{37}$. A nostro avviso, sulla scia del riferimento del notaio, consideriamo che il termine si riferisca alle

\footnotetext{
${ }^{34}$ A. APOSTOLIDIS, op. cit. (n. 32), p. 69 .

35 I testi critici utilizzati per la lista dei prestiti sono quelli di A. APOSTOLIDIS, op. cit. (n. 32), p. 66-68 e quelli di G. COLACICCO, Il regolamento dello scrittorio e della biblioteca di S. Nicola di Casole. Note di Cultura medievale greco-otrantina, tesi di diploma della "Scuola Speciale per Archivisti e Bibliotecari" dell'Università degli Studi di Roma, a.a. 1985/1985, p. 57-61. Per i vari commenti, vd. Colacicco, op. cit., p. 61-67; G. COZZA-LUZI, op. cit. (n. 9), p. 57-64.

${ }^{36}$ G. GIANFREDA, Il monachesimo italo-greco in Otranto, Lecce, 1994 p. 113-116; C. DAQUINO, op. cit. (n. 2), p. 134-135. Il testo critico è quello di A. APOSTOLIDIS, op. cit. (n. 32), p. 66-68, il quale utilizza, per la ricostruzione del testo (p. 66, nota 2), gli appunti di Omont, la copia del Cozza-Luzi e il Codex taurinensis stesso.

${ }^{37}$ Per quanto concerne la localizzazione geografica di Trulatzo (Turlazzo, Trulazzo), l'unica informazione di cui disponiamo è quella di un inventario redatto, il 25 aprile del 1665, dal notaio Carlo Pasanisi. Il documento presenta informazioni molto dettagliate sull'estensione geografica del latifondo monasteriale e sulle sue dipendenze, tra le quali un elenco di un gran numero di chiese e di metochie appartenti al monastero, tra le quali la badia e feudo di Santa Maria di Trulatzo. C. DAQUINO, op. cit. (n. 2), p. 29, citando a sua volta Pasanisi (il testo dell'atto notarile, conservato nel f. 23 presso l'Archivio di Stato di Lecce, viene riprodotto da F. TANZI, L'Archivio di Stato di Lecce. Note e documenti, Lecce, 1902, p. 103), sostiene che l'abbazia e il feudo di S. Maria di Trulatzo, la quale si trovava in territorio otrantino, "era metochia di Vaste (km. 6, 75 da Casole), metochia di Melendugno (km. 21), metochia di Alessano (km. 29) metochia di Castro (km. 14), metochia di Minervino (km. 7), e metochia di Policastro".
} 
dipendenze monastiche, ossia, a quegli edifici satelliti che facevano capo a Casole per la maggior parte delle necessità, tra le quali la biblioteca. Questo tipo di struttura è quello che dalla bizantinistica è stato definito come metochio, una chiesa parrocchiale dipendente da una comunità monastica. Per riuscire a chiarire a livello lessicale la struttura gerarchica tra le due entità, dal momento che non è specificato nel manoscritto, dovremmo intendere per igumeno, in questo caso Nicodemo, a un abate dipendente da un protoigumeno, vale a dire Nicola (detto Nettario), igumeno dell'abbazia matrice, quella di Casole, e da noi conosciuto perché, durante il suo esercizio alla guida del cenobio, venne redattato il typikon.

Tra i prestiti, quelli che destano indubbiamente più curiosità sono quelli relativi a opere profane. Queste, come il manoscritto rivela, venivano consultate unicamente dai laici e il suo prestito veniva autorizzato, come nell'esempio riportato a seguire, dal proprio igumeno:

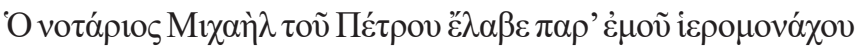

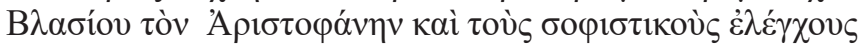

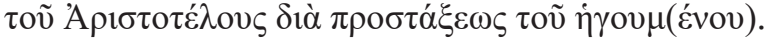

"Il notaio Michele di Pietro, da me, ieromonaco Biagio, ha ricevuto (l'opera di) Aristofane e gli elenchi sofistici di Aristotele ${ }^{38}$ su ordine del igumeno (sic Gianfreda)".

Aristotele risulta essere, in base alla frequenza dei prestiti, un autore molto consultato:

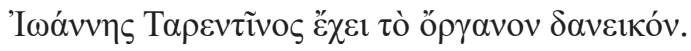

"Giovanni Tarantino ha ricevuto in prestito l'Organon", come anche opere d'altro tipo:

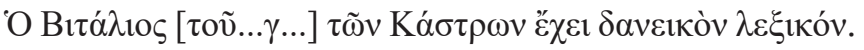

"Vitalio di Castro ha preso in prestito un lessico".

Circa le svariate, ma nel contempo, possibili ipotesi di interpretazione, un esempio molto valido:

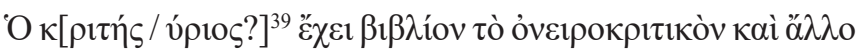

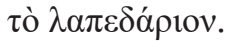

"Il [giudice / signore] ha preso in prestito il libro Sull'interpretazione dei Sogni e un altro, il Lapidario ${ }^{40}$. Un altro esempio ancora:

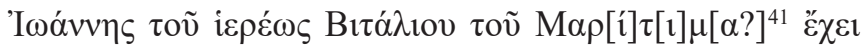

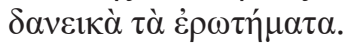

"Giovanni, ha preso in prestito dal sacerdote Vitalio di Marittima gli Erotemata" ${ }^{42}$.

Un ultimo esempio:

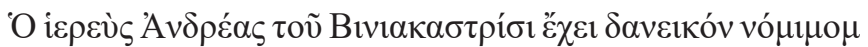
[Omont]; voro... [Cozza-Luzi), [om. Cod. taur.].

"Il sacerdote Andrea di Vignacastrisi ha preso in prestito il Nomocanone 43 (sic Gianfreda, Daquino)". Sempre in ambito laico, un breve appunto, relativo a un notario, nella copia di Cozza-Luzi:

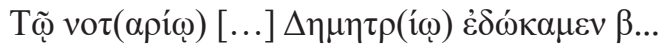

"Abbiamo consegnato al notario Demetrio [...]".

Una striminzita informazione, riferita alla durata di un prestito, ci viene fornita dal manoscritto in questa ricevuta:

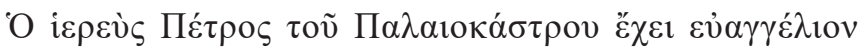

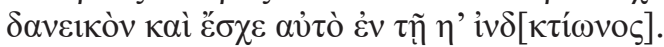

"Il sacerdote Pietro di Paleocastro ha in prestito un Evangeliario e l'ha ricevuto (in prestito) nella settima indizione".

Per quanto riguarda i prestiti di testi religiosi per mano di prelati, il manoscritto fornisce molte più informazioni, sebbene le opere siano abitualmente le stesse e ripetute in varie occasioni. Ciò nonostante, $\mathrm{i}$ frammenti costituiscono, d'altro canto, una fonte di informazioni generosa, la quale ci permette di localizzare con più precisione i limiti del feudo del cenobio casolano ${ }^{44}$.

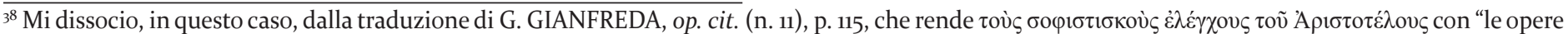
dei Sofisti”, poiché è un chiaro riferimento al titolo originale dell'opera di Aristotele, gli Elenchi sofistici, posizionata nell'Organon subito dopo i Topici.

${ }^{39}$ La lacuna, risolta dal Gianfreda mediante la traduzione "giudice" (крıт́ৎ) è da spiegarsi anche mediante gli altri esempi presenti nel manoscritto, come

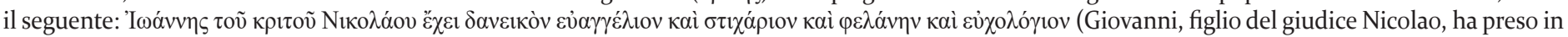

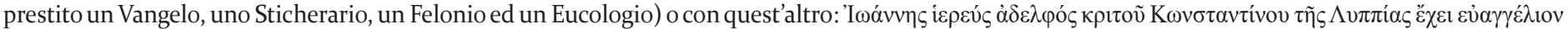
(Il sacerdote Giovanni, fratello del giudice Costantino di Lupiae, ha preso in prestito un vangelo).

$4^{\circ}$ Il Lapidario, trattato medico-magico sulle proprietà delle pietre, presuntamente di origine aristotelica, veniva consultato per le sue informazioni di carattere mineralogico, ma anche, allo stesso tempo, per i riferimenti riguardanti l'astrologia.

${ }^{41}$ La lacuna, a nostro avviso, potrebbe celare un riferimento toponimico, quello della cittadina di Marittima, che rientrerebbe perfettamente all'interno dei limiti del latifondo monasteriale. Tuttavia, considerando la difficoltà di lettura del testo, un'altra congettura potrebbe essere quella relativa al paese di Mapróvos, leggendo la $\mu$ per una v, un riferimento esplicito a un altro comune della Grecia Salentina e all'interno del feudo casolano. G. GIANFREDA, op. cit. (n. 11), p. 115, traduce: "Giovanni del sacerdote Vitalio di Maritem").

${ }^{42}$ Gli Erotemata erano un formato di grammatica greca, scritto in una maniera molto più semplificata, e destinato allo studio della lingua e soprattutto della sua didattica. Tra i più conosciuti, gli Erotemata di Crisolora e quelli di Lascaris, posteriori al manoscritto casolano. Sull'argomento, $v d .$, A. PERTUSI, Erotemata: per la storia e le fonti delle prime grammatiche greche a stampa", in Italia medioevale e umanistica, 5 (1962), p. 329-351.

43 Il Nomocanone era un manuale che raccoglieva le leggi di fonti ecclesiastiche e laiche, falsamente attribuito a Fozio, vd. A. APOSTOLIDIS, op. cit. (n. 32), p. 69.

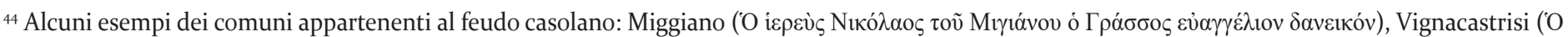

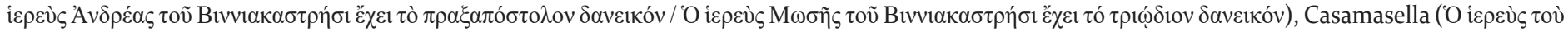

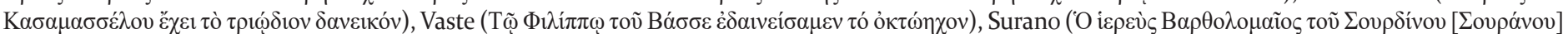

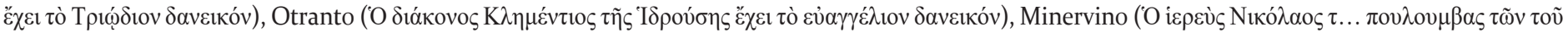

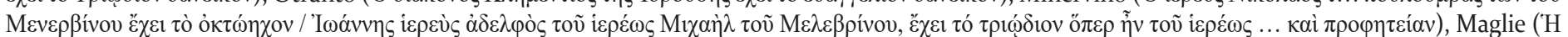




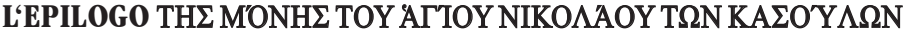

In seguito alla conquista di Otranto del 1480 e la distruzione dell'abbazia, anche la biblioteca andò in fiamme e, di conseguenza, anche i volumi presenti. L'umanista de Ferraris descrive in una sua lettera indirizzata al papa della Rovere l'incendio della biblioteca casulana, dal momento che il pretesto della lettera era quello di offrirgli una copia del Constitutum Constantini, manoscritto facente capo allo scriptorium casulano:

«Nicolaus hydruntinus (...) abbas caenobii (sic) divi Nicolai apud Hydruntum (...) ubi plura ingenii sua monumenta reliquit in dialectica, philosophia et theologia: quae omnia in illa non sine lachrimis memoranda hydruntina clade, monasterio a Turcis direpto et diruto, conflagrarunt simul cum bibliotheca omnis generis librorum, quos ex universa Graecia vir ille magnus congesserat (...) Libellus pervenit ad manus meas ante adventum Turcarum eo tempore, quo versabar cum Stephano archiepiscopo hydruntino consanguineo meo, qui postea gloriosus Christi martyr, una cum universo cleo suo centum fere sacerdotum turcico gladio iugulatus est» 45 [Nicola di Otranto (...) abate del cenobio di San Nicola presso Otranto (...) dove lasciò molte testimonianze del proprio ingegno nella dialettica, nella filosofia e nella teologia: e tutte queste cose, nella famosa strage otrantina, da ricordare non senza lacrime, poiché il monastero era stato saccheggiato e distrutto dai Turchi, bruciarono insieme alla biblioteca ogni genere di libri, che quel grande uomo aveva riunito da tutta la Grecia (...). Il libretto giunse nelle mie mani prima dell'arrivo dei Turchi, all'epoca in cui frequentavo l'arcivescovo Stefano, mio parente, che, in seguito fu martire glorioso di Cristo e che, insieme a tutto il suo clero di quasi cento sacerdoti, fu decapitato dalla scimitarra turca].

Lo studioso leccese attesta, come anche nella testimonianza precedente ${ }^{46}$, il ruolo della biblioteca casulana come centro nevralgico di produzione e diffusione di volumi, e pertanto la sua notorietà: la sua fama era così ampia che, tra i tanti ospiti del monastero, anche il patriarca Bessarione aveva visitato il cenobio casulano e soprattutto il suo scriptorium, dove i calogeri copiavano laboriosamente i preziosi esemplari. Lo studioso Coluccia ricorda, nel suo saggio monografico su Basilio Bessarione ${ }^{47}$, come il cardinale niceno fosse passato personalmente dal monastero di Kasoulon, dal momento che aveva preso parte al capitolo dell'ordine monastico della regola di san Basilio nel novembre 1446 nella chiesa dei Santi Apostoli XII a Roma ${ }^{48}$ e gli era stato affidato direttamente dal papa Eugenio IV il compito di visitare i monasteri siciliani, calabresi e pugliesi, "e riscuotere dagli Abati, dai Commendatori e dai Rettori delle chiese greche, una certa somma, da evolvere ai maestri che istruivano «i monaci e le persone del detto Ordine, e tutti gli altri, chiunque fosse, nella lingua dei Greci, e per il mantenimento degli studenti»" 4 .

La visita del cardinale, tuttavia, era fondamentalmente interessata ai manoscritti del cenobio, in particolar modo a quelli greci, e portò alla sottrazione, da parte del prelato, degli stessi e di altri ancora, i quali, dopo la sua morte, vennero donati al doge di Venezia e passarono a formare parte della collezione della Biblioteca Marciana. Infatti è proprio Bessarione in persona, in sua lettera a Cristoforo Moro ${ }^{50}$, il 31 maggio 1468, a presentare la lista dei manoscritti della sua collezione privata, la quale comprendeva varie casse che pesavano, in media, 200 libbre ciascuna ${ }^{51}$.

\section{CONCLUSIONI}

L'analisi del manoscritto Graecus 216 della Biblioteca di Torino ci ha permesso, dunque, mediante la sua esegesi, di scoprire come fosse la vita di un amanuense bizantino, di come funzionasse uno scriptorium e quali fossero le normative seguite dalla sua biblioteca. Rimane innegabile, per questo motivo, il ruolo decisivo giocato dal cenobio come propulsore di cultura nell'ambito sacro e in quello profano. La finalità dello scriptorium era essenzialmente la diffusione libraia e non l'attività di copiatura fine a sé stessa - con un obiettivo commerciale, ad esempio - bensí orientata a soddisfare la domanda interna del monastero, intendendo per "interno" anche il suo latifondo, e pertanto, le sue strutture satelliti. Una delle necessità di quest'ultime, oltre alla propria formazione culturale, era quella dell'edificazione religiosa e dell'adempimento delle funzioni liturgiche, motivo per il quale la maggioranza dei prestiti risultava essere vincolata a questa necessità.

La notevole importanza di quella che potremmo definire come una "casa editrice casolana" può essere tuttavia apprezzata al giorno d'oggi: infatti, una non piccola parte dei manoscritti infatti, scampata all'incendio e alla distruzione del monastero - ad opera dei Turchi nel 1480, dopo la conquista di Otranto (partem quae superfuerat Turcarum, qui monasterium popolati sunt, bella absumpserunt) - trasportata a Roma presso il patriarca niceno Basilio Bessarione e in seguito, alla sua morte, donata al doge Cristoforo Moro

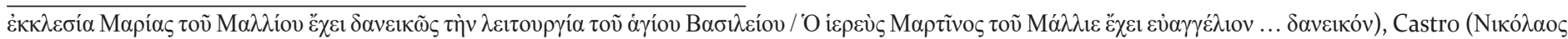

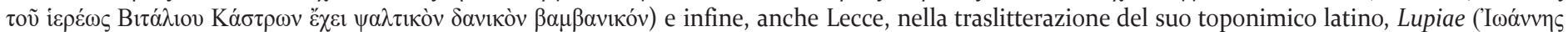

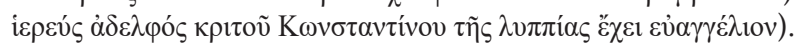

${ }^{45}$ A. DE FERRARIIS GALATEO, Epistole, a cura di A. Altamura, Lecce, 1959 (Scrittori Salentini, 1), p. 181-182, cit. da D. ARNESANO, San Nicola di Casole e la cultura greca in Terra d'Otranto nel Quattrocento, in H. Houben (a cura di), La conquista turca di Otranto (148o) tra storia e mito, Atti del convegno internazionale di studio (Otranto - Muro Leccese, 28-31 marzo 2007), 1, Galatina, 2008, p. 107-140, in particolar modo p. 119, dove l'autore sostiene, alla stessa stregua di H. HOUBEN, Ibid., p. 5-20), la poca affidabilità delle cronache salentine.

${ }^{46} \mathrm{Vd}$. nota 1.

${ }^{47}$ G.L. COLUCCIA, Basilio Bessarione: lo spirito greco e l'Occidente, Firenze, 2009, p. 90-97.

${ }^{48}$ Ibidem, p. 91; G. GIANFREDA, op. cit. (n. 11), p. 136.

49 Ibid., p. 136.

${ }^{50}$ Patrologia graeca, 161, col. 701-714.

${ }^{51}$ C. DAQUINO, op. cit. (n. 2), p. 137, criticando a Rodotà (secondo il quale i volumi donati da Bessarione al doge erano in totale 834 , 533 codici greci, 301 latini), sostiene che in manoscritti erano in totale 746 , dei quali 482 greci e 264 latini. 
(non parva pars Romam ad Bessarionem cardinalem deportata est et inde Venetias) ${ }^{52}$, si disperse successivamente tra le principali biblioteche d'Italia e Europa ${ }^{53}$. I codici casolani, dunque, continuano a svolgere la funzione che era stata loro assegnata sin dal principio: trasmettere e diffondere cultura ai posteri.

${ }_{52}$ A. GALATEO, De situ Iapygiae, 8, 9, op. cit. (n. 1), p. 37.

${ }^{53}$ H. OMONT, L'inventaire des manuscrits grecs et latins donnés à Saint Marc de Venise par le Cardinal Bessarion en 1468, Paris, 1894, p. 21-51; M. PETTA, Codici greci della Puglia trasferiti in biblioteche italiane ed estere, BBGG, 26 (1972), p. 83-129; ID., Codici greci del Salento posseduti in biblioteche italiane ed estere, in Brundisii res, 4 (1972), p. 59-121. 